\title{
Vasospasm and Statin Therapy: Yet Another Cautionary Tale
}

\author{
Alejandro A. Rabinstein
}

Published online: 26 August 2009

(C) Humana Press Inc. 2009

Independent of their cholesterol-lowering effects, statins also have pleiotropic effects which include downregulation inflammation and upregulation of endothelial nitric oxide synthase $[1,2]$. Based on this premise, they have been studied in experimental models of subarachnoid hemorrhage (SAH), which showed that statins might ameliorate the mechanisms involved in the generation of vasospasm [1, 3-5]. Various retrospective cohort studies reported discrepant results: some suggested that statin use the time of SAH was associated with markedly lower risk of vasospasm [6, 7], others found no significant difference in vasospasm risk between statin users and non-users $[8,9]$, and one showed greater risk of vasospasm among statin users, although abrupt statin withdrawal was considered a possible explanation for this finding [10].

Prospective clinical studies soon followed (Table 1). A phase II trial evaluated the role of statins in 80 patients with aneurysmal SAH randomized to receive pravastatin (40 mg daily) or placebo for 14 days [11]. Significant reductions in the incidence of vasospasm (52\% vs. $62 \%$ ) and severe vasospasm $(23.5 \%$ vs. $30 \%)$ defined by TCD were noted among patients treated with pravastatin. The duration of impaired autoregulation was also significantly shorter in the pravastatin group. Vasospasm-related delayed ischemic deficits (DID) occurred in 2 patients in treatment group and 12 patients in placebo group; thereby the incidence of vasospasm-related DIDs was significantly reduced in the pravastatin group by $83 \%$. A subsequent analysis of these investigators showed that there was a

A. A. Rabinstein $(\square)$

Division of Critical Care Neurology, Department of Neurology,

W8B, Mayo Clinic College of Medicine, 200 First Street SW,

Rochester, MN 55905, USA

e-mail: rabinstein.alejandro@mayo.edu strong trend toward better functional outcome at 6 months in the pravastatin group [12]. There was also an $88 \%$ reduction in all-cause mortality; none of the patients in pravastatin-treated group died of vasospasm-related complications, while $12.5 \%$ of deaths in the placebo group were attributed to vasospasm. Physical and psychosocial outcomes at 6 months were better in pravastatin-treated patients on multivariable analysis.

Three other smaller randomized studies have been published with diverse results. Lynch et al. randomized 39 patients to receive simvastatin $80 \mathrm{mg}$ or placebo for 14 days and found a statistically significant reduction of ultrasonographically defined vasospasm in the simvastatin group (26\% vs. $60 \%$ ) [13]. Chou et al. also randomized 39 patients with Fisher grade $3 \mathrm{SAH}$ to simvastatin $80 \mathrm{mg}$ or placebo for up to 21 days, but the results of this study did not show any significant difference between the groups in the rates of vasospasm, vasospasm-related infarcts, or mortality [14]. Although there was a trend toward less vasospasm in the statin group, simvastating-treated patients exhibited a tendency to have worse functional outcomes [14]. Meanwhile, Vergouwen et al. enrolled 32 patients in a randomized study comparing simvastatin $80 \mathrm{mg}$ versus placebo until day 14 after SAH [15]. The main objective of this exploratory study was to investigate the biological effects of statin therapy. Statin-treated patients had reductions in total cholesterol and LDL-cholesterol levels, but were similar to control patients in various parameters of coagulation, fibrinolysis, endothelial function, and inflammation [15]. Statin therapy was safe in all these small trials; specifically, there were no severe cases of myositis or hepatitis reported [11, 13].

The mixed nature of the results of these small prospective randomized, placebo-controlled trials is only one of the arguments for caution. Centers that modified their 
Table 1 Prospective clinical studies evaluating statins in the treatment of aneurysmal subarachnoid hemorrhage

\begin{tabular}{|c|c|c|c|c|}
\hline Study & Design & $\begin{array}{l}\text { Statin type and } \\
\text { dose }\end{array}$ & $\begin{array}{l}\text { Population } \\
\text { size }\end{array}$ & Main results \\
\hline $\begin{array}{l}\text { Tseng et al. }[11, \\
12,21]\end{array}$ & $\begin{array}{l}\text { Randomized, placebo- } \\
\text { controlled }\end{array}$ & $\begin{array}{l}\text { Pravastatin } \\
\quad 40 \mathrm{mg} / \text { day }\end{array}$ & 80 & $\begin{array}{l}\text { Reduced vasospasm } \\
\text { Reduced severe vasospasm } \\
\text { Lower duration of impaired autoregulation } \\
\text { Reduced DID related to spasm } \\
\text { Improved mortality } \\
\text { Improved functional outcome }\end{array}$ \\
\hline Lynch et al. [13] & $\begin{array}{l}\text { Randomized, placebo- } \\
\text { controlled }\end{array}$ & $\begin{array}{l}\text { Simvastatin } \\
\quad 80 \mathrm{mg} / \text { day }\end{array}$ & 39 & $\begin{array}{l}\text { Reduced symptomatic vasospasm } \\
\text { Attenuated serum markers of brain injury }\end{array}$ \\
\hline Chou et al. [14] & $\begin{array}{l}\text { Randomized, placebo- } \\
\text { controlled }\end{array}$ & $\begin{array}{l}\text { Simvastatin } \\
\quad 80 \mathrm{mg}\end{array}$ & 39 & No difference in vasospasm, DCI, or functional outcome \\
\hline $\begin{array}{l}\text { Vergouwen et al. } \\
\text { [15] }\end{array}$ & $\begin{array}{l}\text { Randomized, placebo- } \\
\text { controlled }\end{array}$ & $\begin{array}{l}\text { Simvastatin } \\
\quad 80 \mathrm{mg} / \text { day }\end{array}$ & 32 & $\begin{array}{l}\text { No difference in vasospasm, DCI, or functional outcome } \\
\text { Better lipid panel, but no difference in other laboratory markers }\end{array}$ \\
\hline $\begin{array}{l}\text { Kramer et al. } \\
\text { [17] }\end{array}$ & $\begin{array}{l}\text { Observational, } \\
\text { historical control }\end{array}$ & $\begin{array}{l}\text { Simvastatin } \\
\quad 80 \mathrm{mg} / \text { day }\end{array}$ & 150 & No difference in vasospasm, DCI, or functional outcome \\
\hline $\begin{array}{l}\text { McGirt et al. } \\
\text { [18] }\end{array}$ & $\begin{array}{l}\text { Observational, } \\
\text { historical control }\end{array}$ & $\begin{array}{l}\text { Simvastatin } \\
\quad 80 \mathrm{mg} / \text { day }\end{array}$ & 340 & $\begin{array}{l}\text { No difference in symptomatic spasm, functional outcome, in-hospital } \\
\text { mortality, or LOS }\end{array}$ \\
\hline Kern et al. [16] & $\begin{array}{l}\text { Observational, } \\
\text { historical control }\end{array}$ & $\begin{array}{l}\text { Simvastatin } \\
\quad 40 \mathrm{mg} / \text { day }\end{array}$ & 130 & $\begin{array}{l}\text { No difference in vasospasm, symptomatic spasm, or functional } \\
\text { outcome and mortality at discharge }\end{array}$ \\
\hline
\end{tabular}

$D C I$ delayed ischemic deficits, DCI delayed cerebral infarctions, LOS length of hospital stay

practice and began administering statin therapy to all their patients with SAH after the first reports indicating beneficial effects of statin use on vasospasm have subsequently reported lack of improved outcomes among statin-treated patients compared with historical controls treated at the same institutions before the change in practice [16-18]. Although preliminary data on the use of statins for the prevention of severe vasospasm appear encouraging [19, 20], early promising results must be confirmed on larger, multicenter trials (such as the ongoing STASH trial) before statins can be recommended for this indication.

\section{References}

1. McGirt MJ, Lynch JR, Parra A, Sheng H, Pearlstein RD, Laskowitz DT, et al. Simvastatin increases endothelial nitric oxide synthase and ameliorates cerebral vasospasm resulting from subarachnoid hemorrhage. Stroke. 2002;33(12):2950-6.

2. Wang CY, Liu PY, Liao JK. Pleiotropic effects of statin therapy: molecular mechanisms and clinical results. Trends Mol Med. 2008;14(1):37-44.

3. Cheng G, Wei L, Zhi-Dan S, Shi-Guang Z, Xiang-Zhen L. Atorvastatin ameliorates cerebral vasospasm and early brain injury after subarachnoid hemorrhage and inhibits caspase-dependent apoptosis pathway. BMC Neurosci. 2009;10:7.

4. McGirt MJ, Pradilla G, Legnani FG, Thai QA, Recinos PF, Tamargo RJ, et al. Systemic administration of simvastatin after the onset of experimental subarachnoid hemorrhage attenuates cerebral vasospasm. Neurosurgery. 2006;58(5):945-51.

5. Sugawara T, Ayer R, Jadhav V, Chen W, Tsubokawa T, Zhang JH. Simvastatin attenuation of cerebral vasospasm after subarachnoid hemorrhage in rats via increased phosphorylation of Akt and endothelial nitric oxide synthase. J Neurosci Res. 2008;86(16): 3635-43.

6. Parra A, Kreiter KT, Williams S, Sciacca R, Mack WJ, Naidech AM, et al. Effect of prior statin use on functional outcome and delayed vasospasm after acute aneurysmal subarachnoid hemorrhage: a matched controlled cohort study. Neurosurgery. 2005;56(3):476-84.

7. McGirt MJ, Blessing R, Alexander MJ, Nimjee SM, Woodworth $\mathrm{GF}$, Friedman AH, et al. Risk of cerebral vasopasm after subarachnoid hemorrhage reduced by statin therapy: a multivariate analysis of an institutional experience. J Neurosurg. 2006;105(5): $671-4$.

8. Moskowitz SI, Ahrens C, Provencio JJ, Chow M, Rasmussen PA. Prehemorrhage statin use and the risk of vasospasm after aneurysmal subarachnoid hemorrhage. Surg Neurol. 2009;71(3): 311-7; discussion.

9. Kerz T, Victor A, Beyer C, Trapp I, Heid F, Reisch R. A case control study of statin and magnesium administration in patients after aneurysmal subarachnoid hemorrhage: incidence of delayed cerebral ischemia and mortality. Neurol Res. 2008;30(9):893-7.

10. Singhal AB, Topcuoglu MA, Dorer DJ, Ogilvy CS, Carter BS, Koroshetz WJ. SSRI and statin use increases the risk for vasospasm after subarachnoid hemorrhage. Neurology. 2005;64(6): 1008-13.

11. Tseng MY, Hutchinson PJ, Turner CL, Czosnyka M, Richards H, Pickard JD, et al. Biological effects of acute pravastatin treatment in patients after aneurysmal subarachnoid hemorrhage: a double-blind, placebo-controlled trial. J Neurosurg. 2007;107(6): 1092-100.

12. Tseng MY, Hutchinson PJ, Czosnyka M, Richards H, Pickard JD, Kirkpatrick PJ. Effects of acute pravastatin treatment on intensity of rescue therapy, length of inpatient stay, and 6-month outcome in patients after aneurysmal subarachnoid hemorrhage. Stroke. 2007;38(5):1545-50.

13. Lynch JR, Wang H, McGirt MJ, Floyd J, Friedman AH, Coon $\mathrm{AL}$, et al. Simvastatin reduces vasospasm after aneurysmal 
subarachnoid hemorrhage: results of a pilot randomized clinical trial. Stroke. 2005;36(9):2024-6.

14. Chou SH, Smith EE, Badjatia N, Nogueira RG, Sims JR, Ogilvy $\mathrm{CS}$, et al. A randomized, double-blind, placebo-controlled pilot study of simvastatin in aneurysmal subarachnoid hemorrhage. Stroke. 2008;39(10):2891-3.

15. Vergouwen MD, Meijers JC, Geskus RB, Coert BA, Horn J, Stroes ES et al. Biologic effects of simvastatin in patients with aneurysmal subarachnoid hemorrhage: a double-blind, placebocontrolled randomized trial. J Cereb Blood Flow Metab. 2009.

16. Kern M, Lam MM, Knuckey NW, Lind CR. Statins may not protect against vasospasm in subarachnoid haemorrhage. J Clin Neurosci. 2009;16(4):527-30.

17. Kramer AH, Gurka MJ, Nathan B, Dumont AS, Kassell NF, Bleck TP. Statin use was not associated with less vasospasm or improved outcome after subarachnoid hemorrhage. Neurosurgery. 2008;62(2):422-7.
18. McGirt MJ, Garces Ambrossi GL, Huang J, Tamargo RJ. Simvastatin for the prevention of symptomatic cerebral vasospasm following aneurysmal subarachnoid hemorrhage: a singleinstitution prospective cohort study. J Neurosurg. 2009;110(5): 968-74.

19. Trimble JL, Kockler DR. Statin treatment of cerebral vasospasm after aneurysmal subarachnoid hemorrhage. Ann Pharmacother. 2007;41(12):2019-23.

20. Sillberg VA, Wells GA, Perry JJ. Do statins improve outcomes and reduce the incidence of vasospasm after aneurysmal subarachnoid hemorrhage: a meta-analysis. Stroke. 2008;39(9):2622-6.

21. Tseng MY, Czosnyka M, Richards H, Pickard JD, Kirkpatrick PJ. Effects of acute treatment with pravastatin on cerebral vasospasm, autoregulation, and delayed ischemic deficits after aneurysmal subarachnoid hemorrhage: a phase II randomized placebo-controlled trial. Stroke. 2005;36(8):1627-32. 